\title{
Effect of Porcine Reproductive and Respiratory Syndrome Virus on Porcine Alveolar Macrophage Function as Determined Using Serial Analysis of Gene Expression (SAGE)
}

\author{
L.C. Miller ${ }^{1}$, G.P. Harhay², K.M. Lager', T.P.L. Smith², J.D. Neill ${ }^{1}$ \\ 1 National Animal Disease Center-USDA-ARS, Ames, lowa, USA \\ 2 United States Meat Animal Research Center-USDA-ARS, Clay Center, \\ Nebraska, USA
}

Key words: SAGE, PRRSV, alveolar macrophages

\begin{abstract}
Porcine reproductive and respiratory syndrome virus (PRRSV) is a major pathogen of swine worldwide and causes considerable economic loss. The main target of infection is the porcine alveolar macrophage (PAM). Infection of PAMs by PRRSV causes significant changes in their function by mechanisms that are not understood. We have employed Serial Analysis of Gene Expression (SAGE) to examine the global expression of genes in PRRSV-infected PAMs. Total cellular RNA was prepared from in vitro mock-infected and PRRSV strain VR-2332-infected PAMs at $0,6,12,16$ and 24 hours after infection, and subjected to SAGE analysis to obtain $>100,000$ tags per time point. These sequences were processed to account for sequencing error before generating tag:count lists. These lists were deposited into a modified Identitag database for mapping to porcine and PRRSV genes. Identified unique mRNA tags were analyzed for their identity and relative abundance. Examination of the SAGE data indicated that there were changes in gene expression occurring in the PRRSV-infected PAMs over time post-infection. More than 400 unique tags with significantly altered expression levels were identified $(p<0.01$ with Bonferroni correction). The validity and kinetics of expression of SAGE identified genes were evaluated using real-time RT-PCR.
\end{abstract}

\section{INTRODUCTION}

Porcine reproductive and respiratory syndrome virus (PRRSV), the causative agent of porcine reproductive and respiratory syndrome (PRRS) in swine, is a member 
of the Arteriviridae family in the order Nidovirales. PRRSV causes highly significant losses to the swine industry worldwide [1] as a result of both reproductive failure (late-term abortions and stillbirths) in pregnant sows and respiratory disease (pneumonia) in nursery and grower/finishing pigs [2]. The primary cellular target of PRRSV is the alveolar macrophage of the lung. Thus, porcine alveolar macrophages (PAMs) have a central role in PRRSV infections. Identifying macrophage function specific pathways affected by PRRSV replication can lead to novel gene targets for the control of PRRSV infection. Serial Analysis of Gene Expression (SAGE) is a powerful technique that allows for one to obtain detailed and profound quantitative and qualitative information regarding the gene expression profile, without previous knowledge of the sequence of genes analyzed [3]. SAGE was used to obtain a global picture of gene expression in normal and PRRSV infected PAMs over 24 hours post-infection.

\section{MATERIALS AND MethodS}

Primary PAMs wcre isolated, cultured, and infected, as previously described [4]. Briefly, PAMs were harvested from clinically healthy, PRRS-negative gilts 6-8 weeks of age. Animals were humanely euthanized, following animal care and use protocols, and PAMs harvested under aseptic conditions. Viability of PAMs was determined by trypan blue dye exclusion and cells were tested by PCR for porcine circovirus and Mycoplasma spp. [5,6]. Aliquots of PAMs were frozen and stored in liquid nitrogen until used. Typical yields were $10^{\circ}-10^{\circ}$ PAMs of $>95 \%$ viability. Imınediately prior to use, PAMs were thawed and cultured at $37^{\circ} \mathrm{C}$, $5 \%$ CO2 in Dulbecco's Modified Eagles Media with $5 \%$ foetal bovine serum (FBS; Gibco-Invitrogen, Carlsbad, $\mathrm{CA}$ ) and 1\% antibiotic/antimyotic (Gibco-Invitrogen) for 2 hours. PRRSV strain VR-2332 [7] stock was propagated in MARC-145 cells [8]. To achieve near synchronization of cell infection, flasks containing adherent PAMs were infected at a multiplicity of infection (MOI) of 10 in chilled media and permitted to adhere at $4{ }^{\circ} \mathrm{C}$ for 1 hour to allow for virus binding, but not entry into the cell. After 1 hour, pre-warmed media was added and the cells placed at $37^{\circ} \mathrm{C}, 5 \% \mathrm{CO}_{2}$ with humidification.

Total cellular RNA was prepared from in vitro mock-infected and PRRSV strain VR-2332-infected PAMs at $0,6,12,16$ and 24 hours after infection, and subjected to SAGE analysis [3]. The mRNA was converted to double stranded cDNA with the inclusion of the primer biotin- $5^{\prime}-\mathrm{T}_{18}-3$ ' using the Thermostable RT-PCR System (Invitrogen, Carlsbad, CA). The cDNA was then cleaved with NlallI and the 3'-terminal cDNA fragments were bound and purified using streptavidin-coated magnetic beads (Invitrogen, Carlsbad, CA). Oligonucleotides containing recognition sites for BsmFI were ligated onto the cDNA fragments and the linked cDNA was released from the beads by digestion with $B \cdot \operatorname{sinI}\left(B \operatorname{sinFI}\right.$ cuts DNA 14 bp $3^{\circ}$ of its recognition sequence, thus generating unique sequence tags of $10 \mathrm{bp}$ in length). The released tags were ligated to one another, concatomerized and cloned into the SphI site of the vector pZero 1.0 (Invitrogen, Carlsbad, CA). Colonies were screened by PCR using the M1 3 forward and reverse primers to identify clones containing inserts greater than 300 bp (>25 tags) in length. Clones were ampliticd and sequenced using our high-throughput sequencing pipeline with an ABI 3730 automated sequencer and ABI chemistry (Applied Biosystems Inc., Foster City, CA) at USMARC.

Validation of the results and corroboration of the altered gene expression levels were analyzed by realtime reverse transcription-PCR (RT-PCR). Real-time RT-PCR was done in $25 \mu \mathrm{l}$ reaction volumes using the Invitrogen SYBR green qRT-PCR reaction mix (Invitrogen, Inc., Carlsbad, CA) per supplier's specifications. Primer sequences are available upon request. All primers were used at $100 \mathrm{nM}$ final concentration. PCR cycling conditions were $95^{\circ} \mathrm{C}$ for 15 minutes, and 40 cycles of $94^{\circ} \mathrm{C}$ for 10 seconds, $60^{\circ} \mathrm{C}$ for 30 seconds and $72^{\circ} \mathrm{C}$ for 30 seconds using an Opticon 2 fluorescent thermocycler (MJ Research, Waltham, MA). Analysis of amplification products was done by melt curve where the PCR reactions were heated from 50 to $94 \mathrm{C}$ at a rate of $0.5^{\circ} \mathrm{C} /$ second. For real-time PCR and SAGE tag count comparisons, beta-2-microglobulin $(B 2 M)$ served as the internal control where the number of SAGE tags for $B 2 M$ derived from this library and the amplitication curve from real-time PCR were considered equal and were the basis for comparison of all genes analyzed. To quantify RNA abundance, the comparative threshold cycle $\left(C_{T}\right)$ method was used, which compares the relative amount of target sequence with the reference value chosen and presents the result as relative to the reference value (expression level of mock-infected PAMs). The comparative $C_{\mathrm{T}}$ method $\left(\Delta \Delta C_{\mathrm{T}}\right)$ is the most practical method if the target and reference have similar dynamic ranges [9]. Calculations tor the target transcript quantitation begins with the difference $\left(\Delta C_{T}\right)$ between the $C_{T}$ values of the target and reference. This is followed by transforming these values to absolute values, using the formula: Comparative expression level $=2^{-. .1 . C_{T}}$. 


\section{RESULTS}

Total cellular RNA was prepared from in vitro mock-infected and PRRSV strain VR-2332-infected PAMs at 0,6, 12, 16 and 24 hours post-infection, and subjected to SAGE analysis to obtain $>100,000$ tags per time point. Identified unique mRNA tags were analyzed for their identity and relative abundance. A tag has a quality score (derived from PHRED, a base calling program for DNA sequence traces [10]) and a sequence, ten base pairs in length, that can be used to identify the corresponding transcript. Tags were mapped to transcripts and genes by exact regular expression matching to sequences in GenBank, Harvard Gene Index, Pig Expression Database (Japan), and the USMARC EST databases. Tag abundance was corrected for sequencing error using $R$ and sagenhaft [11]. Relative abundance was calculated based upon the number of times a tag was represented in a given SAGE library [12]. Multidimensional statistical tests [13] were applied to determine which changes in tag abundance were significant. Examination of the preliminary SAGE data indicated that there were changes in gene expression occurring in the PRRSV-infected PAMs based on more than 400 unique tags with significantly altered expression levels identified $(p<0.01$ with Bonferroni correction). SAGE libraries were compared to identify common or differential patterns of expression. Our interest was in expression changes that affect PAMs function; particularly in regard to innate immunity, antigen presentation, and intra- and extracellular signaling.

The SAGE libraries yielded, thus far, 280,903 sequenced tags, which were used to generate a table, representing genes expressed in mock and PRRSV-infected PAMs. The derived catalogue of expressed genes represents a first attempt to generate comprehensive analysis of the PRRSV-infected PAM expression profile (available in Genbank). The wealth of information obtained allowed for the detection of genes involved in normal porcine alveolar macrophage physiology, as well as possible target genes of PRRSV infection. In general, tag frequency in a typical SAGE experiment follows a Gaussian distribution $[14,15]$. Table 1 summarizes the general statistics of these libraries. Tags with a frequency of 1 were not considered for quantitative purposes, because these were likely to represent artefacts of sequencing or of the SAGE procedure [16].

The validity and kinetics of expression of SAGE identified genes were evaluated using real-time RT-PCR (Fig. 1). Control genes that did not respond to PRRSV infection were also included in the analysis as internal controls. Differential expression of the most abundantly expressed tag, corresponding to the ferritin light polypeptide gene $(F T L)$, was confirmed by real-time RT-PCR. The increase in the $F T L$ transcript abundance, as well as a cytochrome P450 (CYP3A) and thioredoxin (TXN), was found to be a result of cell culture. The pro-inflammatory cytokines $I L I A$ and $C C L 4$ declined in transcript abundance in accordance with the findings of Lopez-Fuertes et al. (2000) [17]. Real-time PCR amplification of transcripts known to be upregulated following PRRSV infections $(M X I, R H I V I$, and $U S P)$ showed 40-, 7.5and 14.5-fold increases at 24 hours PI, respectively. The transcripts encoding RNA helicase $R H I V I$ and $M X I$ were induced between 0 and $12 \mathrm{~h}$ post-PRRSV-infection in accordance with Zhang et al. (1999) [18]. 
Table 1: Summary of SAGE analysis of transcripts dysregulated by PRRSV.

\begin{tabular}{|cccccc|}
\hline & \multicolumn{2}{c}{ SAGE Tags } & & \\
\hline Library $^{\mathrm{a}}$ & Total & Unique & $\begin{array}{r}\text { Known } \\
\text { Transcripts }\end{array}$ & $\begin{array}{c}\text { Unknown } \\
\text { Transcripts }\end{array}$ & No Match \\
\hline T0 Mock & 46,421 & 13,583 & $11832(25 \%)$ & $5031(11 \%)$ & $29557(64 \%)$ \\
\hline T6 & 66,486 & 23,547 & $17110(26 \%)$ & $5277(8 \%)$ & $44094(66 \%)$ \\
\hline T12 & 66,194 & 26,187 & $15458(23 \%)$ & $4977(8 \%)$ & $45759(69 \%)$ \\
\hline T16 & 36,446 & 13,650 & $9244(25 \%)$ & $2254(6 \%)$ & $24949(68 \%)$ \\
\hline T24 & 65,362 & 18,668 & $16687(26 \%)$ & $5812(9 \%)$ & $42863(66 \%)$ \\
\hline Total & 280,903 & 95,604 & $70331(25 \%)$ & $23351(8 \%)$ & $187222(67 \%)$ \\
\hline
\end{tabular}

${ }^{a}$ The librarics are T0 Mock, a total SAGE tag count of porcine alveolar macrophages mock-infected; T6, a total SAGE count of porcine alveolar macrophages infectcd with PRRSV 6 hours post-infection; T 12, a total SAGE count of porcine alveolar macrophages infected with PRRSV 12 hours post-infection; T16, a total SAGE count of porcine alveolar macrophages infected with PRRSV 16 hours post-infection; and T24, a total SAGE count of porcine alveolar macrophages infected with PRRSV 24 hours post-infection.

\section{Discussion}

SAGE libraries were generated defining the transcription of non-infected PAMs and PAMs-infected with PRRSV that will be a publicly available resource. Macrophage transcript abundance that varied in correlation with PRRSV replication was identified. This research may identify genes that respond to specific stages of the virus life cycle such as virus binding and entry versus replication. Responding genes were identified that have not yet been represented in sequence databases. We periodically BLAST these sequences against sequence databases until matches are identified. Further study of the genes, their transcript abundance, protein level, and protein function will enhance our understanding of the interaction of PRRSV with the porcine macrophage. Possible outcomes may include the identification of virulence mechanisms, the development of next generation diagnostic assays and more rational vaccine design to more effectively limit viral replication and spread.

\section{ACKNOWLEDGEMENTS}

The authors would like to acknowledge Drs. M. E. Kehrli, Jr. and T. L. Nicholson for helpful discussions and reviewing of the manuscript. We would like to thank W. Boatwright, S. Pohl and R. Steeves for technical assistance, and $\mathrm{S}$. Ohlendorf for secretarial assistance in preparation of the manuscript. This work was supported in part by a grant from the National Pork Board to J.N. and L.M. (06-122).

Mention of trade names or commercial products in this article is solely for the purpose of providing specific information and does not imply recommendation or endorsement by the U.S. Department of Agriculture. 


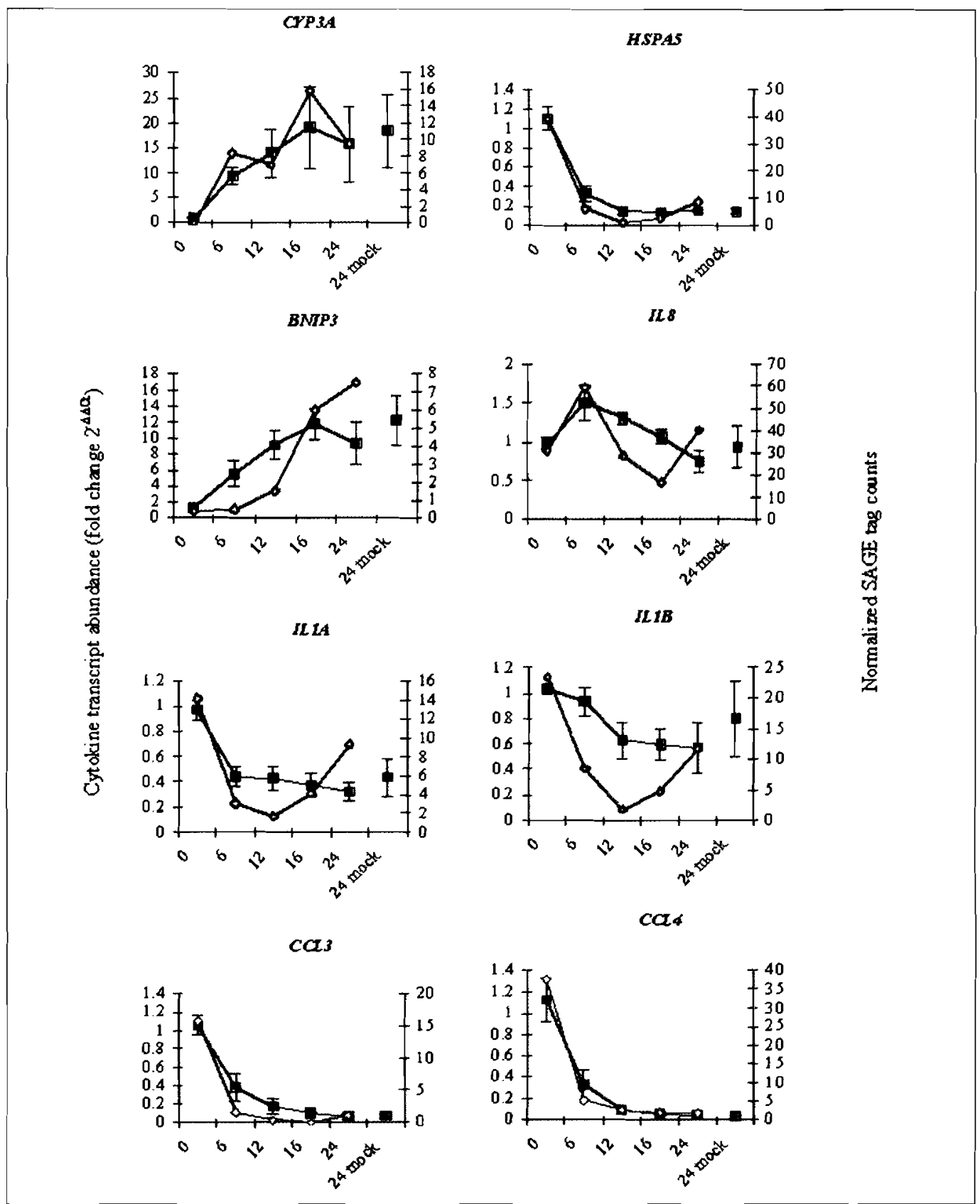

Fig. 1: Real-time RT-PCR validation of SAGE results.

Validation by real-time RT-PCR of RNA from the in vitro PRRSV-infected PAM samples used in the SAGE analysis of selected transcripts showing differential expression. Real-time RT-PCR transcript abundance results (filled squares; left $y$ axis) and SAGE tag counts normalized to total tags per library (open diamonds; right $y$ axis) are shown for transcripts: Cytochrome P450 (CYP3A; TC281236); heat shock 70kDa protein 5 (HSPA5; BW956325); BCL2/adenovirus E1B $19 \mathrm{kDa}$ interacting protein 3 (BNIP3; AK240169); interleukin-8 (ILS; NM 213867); interleukin 1, alpha (ILIA; NM_214029); interleukin I, beta (ILIB: NM_214055); chemokine (C-C motif) ligand 3 (CCl.3; NM_00 009579 ); chemokine (C-C motif) ligand 4 (CCL4; TC28879l). The '24 mock' result shows the real-time RTPCR fold change in transcript abundance as a result of being in cell culture. 


\section{REFERENCES}

1 Rossow KD: Porcine reproductive and respiratory syndrome. Vet Pathol 1998;35(1):1-20.

2 Zimmerman JJ, Yoon KJ, Wills RW, Swenson SL: General overview of PRRSV: a perspective from the United States. Vet Microbiol 1997;55(1-4):187-196.

3 Velculescu VE, Zhang L, Vogelstein B, Kinzler KW: Serial analysis of gene expression. Science $1995 ; 270: 484-487$.

4 Chiou MT, Jeng CR, Chueh LL, Cheng CH, Pang VF: Effects of porcine reproductive and respiratory syndrome virus (isolate tw91) on porcine alveolar macrophages in vitro [In Process Citation ]. Vet Microbiol 2000;71(1-2):9-25.

5 Opriessnig T, Yu S, Gallup JM, Evans RB, Fenaux M, Pallares F, et al: Effect of vaccination with selective bacterins on conventional pigs infected with type 2 porcine circovirus. Vet Pathol 2003:40(5):521-529.

6 Stakenborg T, Vicca J, Butaye P, Imberechts H. Peeters J, De Kruif A, et al: A multiplex PCR to identify porcine mycoplasinas present in broth cultures. Vet Res Commun 2006;30(3):239-247.

7 Allende R, Kutish GF, Laegreid W, Lu Z, Lewis TL, Rock DL, et al: Mutations in the genome of porcine reproductive and respiratory syndrome virus responsible for the attenuation phenolype. Arch Virol 2000;145(6):1149-1161.

8 Kim HS, Kwang J, Yoon IJ, Joo HS, Frey ML: Enlanced replication of porcine reproductive and respiratory syndrome (PRRS) virus in a homogeneous subpopulation of MA-104 cell line. Arch Virol $1993 ; 133(3-4): 477-483$.

9 Pfaffl MW: A new mathematical model for relative quantification in real-time RT-PCR. Nucleic Acids Res 2001;29(9):e45.

10 Ewing B, Green P: Base-calling of automated sequencer traces using phred. II. Error probabilities. Genome Res. 1998;8(3): 186-194.

11 Beissbarth T, Hyde L, Smyth GK, Job C, Boon WM, Tan SS, et al: Statistical modeling of sequencing errors in SAGE libraries. Bioinformatics 2004;20 Suppl 1:131-39.

12 Madden SL, Wang CJ, Landes G: Serial analysis of gene expression: from gene discovery to target identification. Drug Discov Today 2000;5(9):415-425.

13 Romualdi C, Bortoluzzi S, D'Alessi F, Danieli GA: IDEG6: a web tool for detection of differentially expressed genes in multiple tag sampling experiments. Physiol Genomics 2003;12(2):159-162.

14 Audic S, Claverie JM: The significance of digital gene expression profiles. Genome Res 1997;7( I0):986-995.

15 Ruijter JM, Van Kampen AH, Baas F: Statistical evaluation of SAGE libraries: consequences for experimental design. Physiol Genomics 2002:11(2):37-44.

16 Yamamoto M, Wakatsuki T, Hada A. Ryo A: Use of serial analysis of gene expression (SAGE) technology. J Immunol Methods 2001:250(1-2):45-66.

17 Lopez-Fuertes L, Campos E, Domenech N, Ezquerra A, Castro JM, Dominguez J, et al: Porcine reproductive and respiratory syndrome (PRRS) virus down-modulates TNF-alpha production in infected macrophages. Virus Res. 2000;69(1):41-46.

18 Zhang X, Shin J, Molitor TW, Schook LB, Rutherford MS: Molecular responses of macrophages to porcine reproductive and respiratory syndrome virus infection. Virology. 1999;262(1):152-162.

Dr. Laura C. Miller, Virus and Prion Diseases of Livestock Research Unit, National Animal Disease Center, Agricultural Research Service, USDA, P.O. Box 70, 2300 Dayton Avenue, Ames, IA 50010, USA.

E-mail: laura.miller@ars.usda.gov 